\section{Intestinale Obstruktion als Spätkomplikation}

\begin{abstract}
Die Liste der Nachwirkungen maligner Erkrankungen und ihrer Therapien im Kindesalter ist lang, noch als Erwachsene haben die Betroffenen damit zu tun. Muss man auch mit intestinalen Obstruktionen als Komplikation rechnen?
\end{abstract}

$\mathrm{D}$ ie Frage wurde bei 12.316 Personen der Childhood Cancer Survivor Study (CCSS), die mindestens 5 Jahre nach Diagnose einer Krebserkrankung im Kindesalter noch am Leben waren, und 4.023 Geschwistern untersucht. Bei 2.002 der Krebsüberlebenden hatte sich der Tumor im Abdomen oder Becken befunden (Lymphom, Neuroblastom, Wilms-Tumor, Knochentumor, Weichteilsarkom), bei den anderen nicht (vor allem Leukämien, ZNS-Tumoren). 77\% der Überlebenden mit Tumor in Abdomen oder Becken unterzogen sich innerhalb der ersten 5 Jahre nach Diagnose mindestens einer Operation in diesem
Bereich, von den anderen nur $10 \%$. Ermittelt wurde die kumulative Inzidenz später ( $\geq 5$ Jahre nach Diagnose) intestinaler Obstruktionen mit Indikation zur Operation (IOS), wobei Zweitmalignome, spätes Rezidiv und Tod als konkurrierende Risiken berücksichtigt wurden.

165 Überlebende entwickelten eine späte IOS. Sie waren zu diesem Zeitpunkt median 19 Jahre alt (5-50 Jahre), seit der Diagnose waren median 13 Jahre vergangen. Auch 14 Geschwister erkrankten an einer IOS. Die kumulative Inzidenz einer späten IOS (bis zu 35 Jahre nach Krebsdiagnose) betrug bei einem Tumor im Abdomen oder Becken 5,8\% (95\%-Konfi- denzintervall [95\%-KI] 4,4-7,3\%), bei einer anderen Krebserkrankung 1,0\% (95\%-KI 0,7-1,4\%) und unter den Geschwistern 0,3\% (95\%-KI 0,1-0,5\%). Ein Tumor im Abdomen oder Becken und eine Radiotherapie in diesem Bereich innerhalb von 5 Jahren nach Krebsdiagnose erhöhten die Rate später IOS, die Chemotherapie hatte keinen Einfluss. Eine späte IOS erhöhte die Mortalität der Krebsüberlebenden (adjustierte Rate Ratio $1,8,95 \%$-KI $1,1-2,9 ; \mathrm{p}<0,016)$.

Fazit: Nach einer Krebserkrankung im Kindesalter, v.a. in Abdomen oder Becken, besteht lange ein erhöhtes Risiko für eine IOS. Da sie auch die Mortalität steigert, ist erhöhte Aufmerksamkeit geboten.

Kathrin von Kieseritzky

Madenci AL et al. Intestinal Obstruction in Survivors of Childhood Cancer: A Report From the Childhood Cancer Survivor Study. J Clin Oncol. 2015;33(26):2893-900.

\section{Vorhersage schwerer Fluoropyrimidintoxizitäten}

\section{Die Dihydropyrimidin-Dehydrogenase (DPD) ist das entscheidende Enzym für den Fluoropyrimidin(FL)-Abbau. Varianten in DPYD, dem für DPD kodieren- den Gen, können zu einer geringeren Enzymaktivität und so zu schwererer Toxizität führen. Daher wird eine prätherapeutische pharmakogenetische Testung empfohlen, die sich aber in der Klinik bisher nicht durchgesetzt hat.}

$\mathrm{n}$ einer Studie wurden die Proben von 603 Patienten, die FL erhalten hatten, retrospektiv auf das Vorhandensein von 8 SNPs (,single nucelotid polymor-

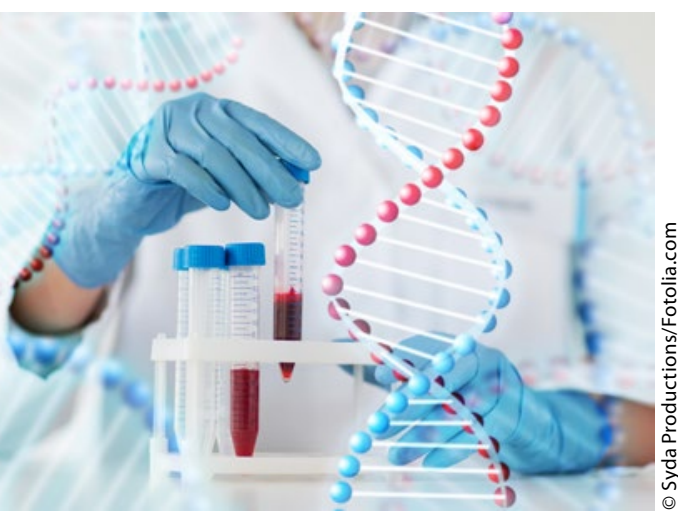

Ein Gentest vor der FluoropyrimidinTherapie warnt vor schwerer Toxizität. phisms") im DPYD-Gen getestet und ihre Assoziation mit einer Toxizität $\geq$ Grad 3 untersucht, die innerhalb der ersten 3 Behandlungszyklen aufgetreten sein musste. Ein Test, der 3 dieser SNPs erfasst (rs3918290, rs55886062 und rs67376798), steht zur prätherapeutischen Untersuchung zur Verfügung (3-Marker-Test). Hinweise aus der Literatur legen nahe, dass 5 weitere Polymorphismen (rs2297595, rs1801160, rs1801158, rs1801159 und rs17376848) ebenfalls relevant sein könnten.

18 der 603 Patienten $(3,0 \%)$ trugen mindestens 1 variantes Allel, und 11 davon $(61,1 \%)$ entwickelten eine Toxizität $\geq$ Grad 3. Ein Patient wies 2 SNPs auf (rs3918290 und rs55886062), er verstarb aufgrund der FL-Toxizität. Von den $7 \mathrm{~Pa}-$ tienten mit einem der ersten 3 Marker ohne Toxizität $\geq$ Grad 3 benötigten $57 \%$ eine Dosis- oder Protokoll-Modifikation der FL wegen mäßiger chronischer Toxizität. rs3918290 und rs67376798 erwiesen sich in der Berechnung mit 2 verschiedenen statistischen Verfahren jeweils als signifikant mit einer Toxizität $\geq$ Grad 3 assoziiert $(p=0,003 ; p=0,048)$. Die Assoziation mit dem 3. Polymorphismus, rs55886062, war nicht signifikant - wohl aufgrund seiner Seltenheit. Dennoch ist auch er offenbar klinisch relevant. Denn einer der beiden betroffenen Patienten verstarb schon nach dem ersten Zyklus aufgrund der Toxizität. Keiner der anderen, zusätzlich getesteten, 5 SNPs war mit starker Toxizität assoziiert. Insgesamt ergab sich für die ersten 3 Marker eine TestSpezifität von $99 \%$, allerdings bei niedriger Sensitivität.

Fazit: Die Ergebnisse bestätigen die klinische Validität und Spezifität der prätherapeutischen Testung auf 3 DPYD-SNPs zur Vermeidung schwerer Toxizitäten durch FL. Dies spricht für ihren Einsatz in der klinischen Onkologie. Brigitte Schalhorn

Toffoli $\mathrm{G}$ et al. Clinical validity of a DPYD-based pharmacogenetic test to predict severe toxicity to fluoropyrimidines. Int J Cancer. 2015;137(12): 2971-80. 